\title{
Publisher Correction: Gut feelings: microRNAs tune protein quality control and ageing to odours
}

Rachel Kaletsky and Coleen T. Murphy

Correction to: Nature Metabolism https://doi.org/10.1038/s42255-019-0042-y, published online 18 February 2019.

In the version of the article originally published, the study by Finger et al. was incorrectly described in one sentence in the third paragraph as implicating the microRNA mir-73 in the regulation of proteostasis; mir-71 was the correct microRNA. The error has been corrected in the HTML and PDF versions of the article.

Published online: 6 March 2019

https://doi.org/10.1038/s42255-019-0050-y 